\title{
SEQUENTIAL IN-VEHICLE GLANCE ANAL YSIS OF ATTENTION MAINTENANCE BEHAVIOR FOR TRAINED AND UNTRAINED YOUNG DRIVERS
}

\author{
Pınar Bıçaksız ${ }^{1}$, Dakota B. Palmer ${ }^{1}$, Yusuke Yamani ${ }^{1}$, Siby Samuel ${ }^{2}$ \\ ${ }^{1}$ Department of Psychology, Old Dominion University \\ ${ }^{2}$ Department of Mechanical and Industrial Engineering, University of Massachusetts Amherst \\ Email: pbicaksiz@gmail.com
}

\begin{abstract}
Summary. In-vehicle tasks often require drivers to glance away from the forward roadway while driving for relatively long durations. Long in-vehicle glances are associated with an elevated risk of fatal crash involvement. In the current effort, the glance data reported in Yamani et al. (2016) where drivers received either an integrative training program for higher cognitive skills including attention maintenance (SAFE-T) or a placebo program, have been reanalyzed to uncover differences in glance behavior among trained and untrained drivers. By applying two alternative analytic techniques, the summed excess glance durations and the sequential glance analysis, the data indicated that the training was especially effective on limiting the duration of the first glance in the sequence compared to the rest of the glances in the sequence. The results imply that the training program has differential impacts on attention maintenance behavior at different stages of task interaction, such as orienting to, and processing of task-relevant information.
\end{abstract}

\section{INTRODUCTION}

The high accident risk of novice drivers is one of the most consistently supported findings in road safety literature (Bjørnskau \& Sagberg, 2005). Crash statistics per mile driven show that 16year-old novice drivers have non-fatal crash rates thrice than that of 18-year-old drivers and ten times that of adults (McKnight \& McKnight, 2003). This overrepresentation of young drivers in crashes may be attributed to immaturity and risk taking tendencies associated with young age as well as inadequate driving skills due to inexperience (Shinar, 2007) leading to poor visual search and improper allocation of attention to the forward roadway (McKnight \& McKnight, 2003).

Although driving by itself demands a myriad of perceptual-cognitive and motor processes, drivers often engage in various in-vehicle tasks such as conversations via cell-phones (Drews \& Strayer, 2008) and the use of smartphone applications (McDonald \& Sommers, 2015), detracting their attention away from the forward roadway (Klauer, Dingus, Neale, Sudweeks, \& Ramsey, 2006). Driver distraction involves a temporary shift of attention from driving by focusing on stimuli unrelated to the immediate driving environment such as objects, people or tasks (Hedlund, Simpson, \& Mayhew, 2005), often coupled with eye movements (Pollatsek, Divekar, $\&$ Fisher, 2013). Especially long glances away from the roadway longer than 2 seconds increase on-road fatal crash risk by four times as much as baseline condition (Klauer et al., 2006; Summala, Lambe, \& Laakso, 1998). The real-world impact of driver distraction thus cannot be overstated (Green, 2002; Horrey, 2009).

To remediate the adverse effects of especially long in-vehicle glances during driving, training programs have been designed for drivers to limit their in-vehicle glance durations. One such intervention, FOrward Concentration and Attention Learning (FOCAL; Divekar et al., 2013; 
Pradhan et al., 2009, 2010, 2011), aims to train drivers to limit each in-vehicle glance duration to no longer than 2 seconds when performing in-vehicle tasks while driving. Driving simulator (Pradhan et al., 2009, 2010) and on-road evaluation studies (Pradhan et al., 2011) confirmed the effectiveness of FOCAL in reducing the overall proportion of the especially long in-vehicle glances while driving, in turn maintaining their attention on the immediate forward roadway. The longitudinal evaluation study on a driving simulator also supported the effectiveness of FOCAL at reducing the proportion of long in-vehicle glances for up to four months after training (Divekar et al., 2016).

Recently, two measures have been proposed to quantify drivers' attention maintenance behavior more rigorously. First, Pollatsek and colleagues (2013) proposed the summed excess glance durations for assessing in-vehicle glance behavior when constrained to a threshold at a certain time interval. Specifically, in this measure, an excess glance duration score for each in-vehicle glance is defined as:

1. If glance duration $\leq$ threshold, then excess glance duration $=0$, else

2. If glance duration $>$ threshold, then excess glance duration $=$ glance duration - threshold. Then, for each driver, the sum of excess glance duration scores is computed. This measure thus provides an estimate of the likelihood of crash by weighting on in-vehicle glance durations longer than a threshold (e.g., 2.0 seconds), assuming that glances longer than the threshold are riskier than shorter ones.

Second, Yamani and colleagues (2015) proposed sequential in-vehicle glance distributions that allow quantification of changes in drivers' in-vehicle glancing behavior across successive glances. In-vehicle tasks can demand not only the orientation of attention (e.g., turning on the headlight) but also the subsequent processing of visual information (e.g., reading a map), thus predicting that as the visual demand increases particularly later in a sequence, the drivers are more likely to execute especially long in-vehicle glances. The results demonstrated that the first and second glances in the sequence were in fact statistically shorter than subsequent glances, indicating that especially long in-vehicle glances do arise later in a sequence when visual demand becomes greater in certain tasks such as, reading a map and dialing a phone number.

The present study aims to apply the two methods of analyzing in-vehicle glance behaviors to data obtained from young drivers trained either on an integrated program (SAFE-T; Hamid, Samuel, Borowsky, Horrey \& Fisher, 2016; Yamani, Samuel, Knodler, \& Fisher, 2016) or a placebo program, and examine how the training program influences the drivers' glancing behaviors while driving. Briefly, SAFE-T is a PC-based integrated training program targeting three higher cognitive skills, hazard anticipation (Pradhan et al., 2005), hazard mitigation (Muttart et al., 2013, 2014) and attention maintenance (Pradhan et al., 2009), and proven effective in improving all the three skills on a driving simulator study (Yamani et al., 2016). Of current interest is the component of attention maintenance (AM) in SAFE-T that can be administered in approximately one third of the time required for FOCAL. We hypothesize that, compared to untrained drivers, trained drivers 1) show lower summed excess glance duration scores and 2) execute shorter in-vehicle glances than untrained drivers, more likely at earlier times in a sequence for each task. To this end, the available data set published in Yamani et al. (2016) were reanalyzed in the current effort. 


\section{METHOD}

In the Yamani et al. (2016) study, 32 young drivers (16 males and 16 females; $M=17.95$ years, range $=16-18$ years) with a junior operator's license were randomly assigned to a SAFE-T $(N=16,7$ males and 9 females) or a placebo $(N=16,9$ males and 7 females $)$ group. The experiment was conducted on a medium-fidelity driving simulator (STISIM, System Technology, Inc.) consisting of three 60" screens, three projectors, a sound system, three computers controlling graphic images, and a built-up cab with an adjustable driver's seat, a steering wheel, accelerator and brake pedals. There were four attention maintenance evaluation scenarios consisting of two-lane roads with no surrounding traffic, no lead vehicle events or any other kind of potential or actual hazards. The participants were instructed to perform four AM tasks while driving on the simulator; searching for a $\mathrm{CD}$, depositing change, dialing a number and locating a street on a map. They were given auditory instruction to start a task and $15 \mathrm{~s}$ later, a short beep sound indicated the end of the task. The tasks started at predetermined locations on the roadway in each scenario.

Participants' glance durations and scanning behaviors were tracked by a monocular eye-tracker (ASL Mobile Eye, Applied Science Laboratories, Inc.) at a sampling rate of $30 \mathrm{~Hz}$. The system software integrated the eye images into the recorded scene view in a single video with a superimposed crosshair indicating the gaze locations in each frame.

The attention maintenance segment of the SAFE-T program instructed the participants that they should keep their in-vehicle glances shorter than $2 \mathrm{~s}$ if they must glance away from the forward roadway. The training itself involved the participants viewing either a display of a street map or a video of a forward roadway environment, but never both at the same time. The program began with a view of the roadway and participants used two buttons to switch back and forth between a view of the forward roadway (DRIVE) and the view of a map (MAP). The driver was provided with 20 seconds to identify a target street (cross-street) on the map, one which intersects the street that the participant was traveling in the scenario. If the glance at the map exceeded two seconds, the participant would hear a beep. In each scenario (4 in total), the participant was given two separate trials to finish the map task. To successfully complete the task, the driver had to have no single glance greater than two seconds at the map. The participant was then asked to enter the name of the cross street. Overall training score incurred a penalty for each time the street name was incorrectly identified by the participant. After each of the four AM scenarios, participants were presented with error feedback and a chance to master the skill. The placebo training assigned to participants in the control group contained a video discussion of simple driver skills such as changing tires, measuring tire pressure, and information on motor oil, none of which directly or indirectly related to improving drivers' attention maintenance ability.

After the completion of the assigned training program, participants navigated a practice drive in the simulator to get acquainted with the simulator and the AM tasks. They were then outfitted with an eye tracker and asked to drive the experimental scenario set on the simulator. Participants were instructed to drive as they generally do on an actual road in accordance with all the traffic rules, signs and speed limits. 


\section{RESULTS}

The duration of a single in-vehicle glance was defined as the time interval from when a driver's eyes leave the forward roadway up until the gaze is returned to the forward roadway (Pradhan et al., 2011). Due to the data storage problem, data of 6 drivers were lost. The remaining data from 10 drivers in the untrained group and 14 drivers in the trained group were analyzed in this paper. Two independent raters coded the time points at which the participant's gaze leaves the forward roadway and returns to the forward roadway during the 15 seconds corresponding to the invehicle task. If two raters disagreed, they discussed such glances and resolved the disagreement. In total, 546 glances for the trained drivers $(\mathrm{M}=1.08 \mathrm{~s}, \mathrm{SD}=.73$, range $=.03-4.21)$ and 389 glances for the untrained drivers $(\mathrm{M}=1.13 \mathrm{~s}, \mathrm{SD}=.72$, range $=.03-4.41)$ were analyzed for understanding the patterns of in-vehicle glance behavior.

Summed excess glance durations. Excess glance duration scores were calculated for each participant using the equations above with 2-second and 1.5-second thresholds (Pollatsek et al., 2013) and submitted to independent-samples t-tests separately. With both 2-second and 1.5second thresholds, the trained drivers produced numerically shorter excess glance durations than the untrained drivers, but the difference was not statistically different $[\mathrm{M}=.63$ vs. .58 seconds with 2 -second threshold and $\mathrm{M}=1.49$ vs. 1.36 seconds with 1.5 -second threshold; both $t \mathrm{~s}<1]$. Sequential glance distributions. Following the technique used in Yamani et al. (2015), we limited our sequential analysis to the first seven glances. Figure 1 presents the complementary cumulative distribution function (CDF) for each of the seven successive glances, collapsed across participants. The complementary CDF in the current context indicates the probability that the glance duration will be greater than or equal to a time, $t$. Visual inspection of the data indicates short glances for the first two glances in the sequence compared to the rest for both, the trained and the untrained driver groups. We conducted a series of the Kolmogorov-Smirnov (K$\mathrm{S})$ tests with Bonferroni correction to examine whether samples in each glance sequence arise from different distributions. After the Bonferroni correction, the critical alpha value was corrected to .0025 . The results partly corroborated with the results of the visual inspections. For the untrained group, the data did not provide any reliable evidence that the samples of the first two glances in a sequence arise from a distribution different than that of the samples of later glances [all $p \mathrm{~s}>.004$ ]. For the trained group, a distribution of the first glance was statistically different from the 4 th and the 6 th glances in the sequence $[p=.001$ and $p=.002]$. The rest of the comparisons were not statistically reliable [all $p \mathrm{~s}>.021]$.
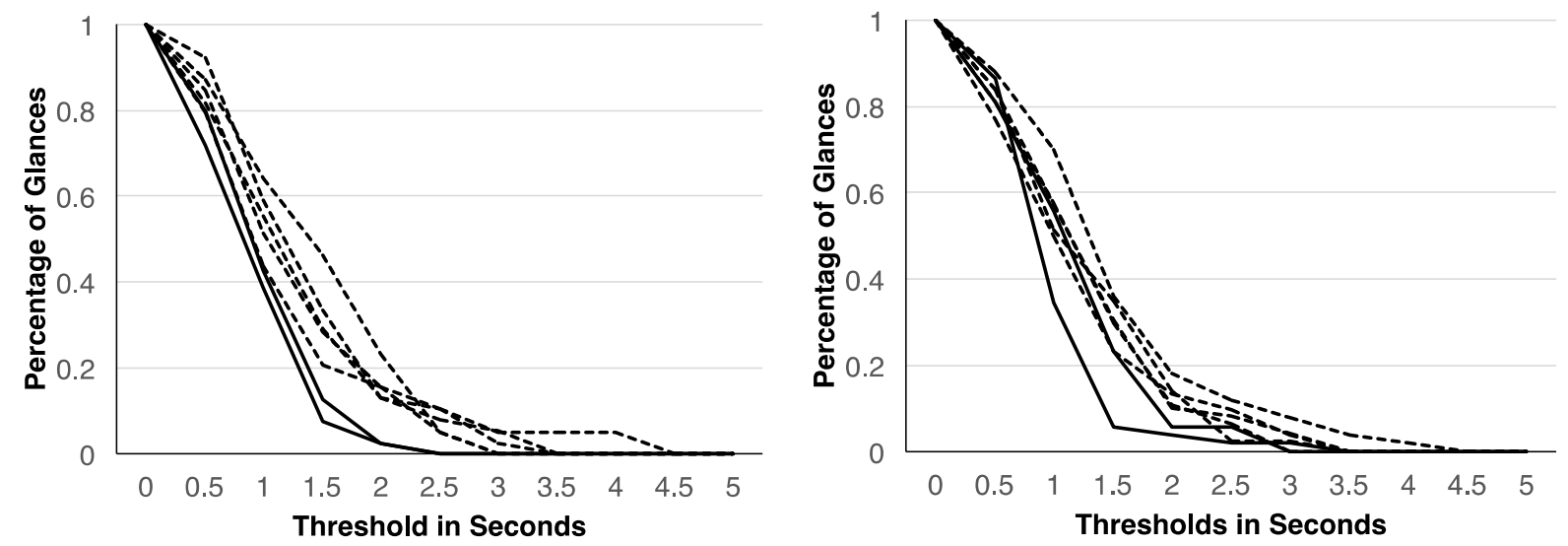

Figure 1. Distribution of early (first and second glances; solid) and later (third to the seventh glances; dashed) in-vehicle glances for placebo (left) and SAFE-T (right) groups. 


\section{DISCUSSION}

The present study employed two newly proposed techniques to reanalyze the in-vehicle glance data in Yamani et al. (2016). First, the analysis of the summed excess glance durations (Pollatsek et al., 2013) did not show reliable differences in the especially long glance durations between the trained and untrained groups. Second, and importantly, the results of the sequential glance distribution analysis suggest that 1) early glances in a sequence tend to be shorter than later ones in general, and 2) the attention maintenance training is most effective for limiting the durations of early glances within a sequence.

Yamani and colleagues (2015) performed a task analysis to quantify visual demands of the invehicle tasks. They found that proportions of glances longer than two seconds dramatically increased when the visual demand of driving was higher, and this increase was more prominent later in a sequence of glances than earlier. One account for this data pattern is that drivers execute shorter glances when a task requires orienting of attention to an object for performing a task (e.g., locating and holding a smartphone) while they require longer glances when the task demands some level of processing of visual information (e.g., finding an app on the smartphone display). Literature examining task complexity reports several interaction characteristics (Horrey, Wickens \& Consalus, 2006). Along this line of thought, the current findings may imply that the SAFE-T training program effectively reduced the duration of the first in-vehicle glance that is likely to support the orienting phase of the task interaction (Yamani et al., 2015).

We still need to answer why trained drivers are still more likely to execute glances longer than 2 seconds later than earlier in a sequence of glances while driving. Perhaps after they orient their attention to the task, the drivers might have allocated resources to perform the task but less so to monitor each glance duration, because the attention maintenance skill has not yet been completely automatized. Tasks that have not been automatized are subject to a greater cost of multi-tasking (Schneider \& Shiffrin, 1977). While glancing into the vehicle, trained drivers were more likely to both perform the in-vehicle task and monitor their in-vehicle glance durations, a form of multitasking, which might have led to a relatively lower effect of the training on glances that occur later in a sequence.

There are a few limitations with the present study. First, we were unable to analyze the complete dataset from Yamani et al. (2016). Second, the small number of data points here did not allow further analyses of in-vehicle glancing behavior across different task structures. Tasks that involve greater levels of visual demands, for example, are shown to increase proportions of especially long in-vehicle glances (Yamani et al., 2015). Third, the current results may not necessarily generalize to real-world behaviors on road. Fourth, we still need to determine whether the currently observed patterns in sequential glance durations persist for a longer period of time after AM training. Future research should address these limitations and more closely examine relationships between the effect of the existing training and drivers' ability to control attention in tasks requiring different levels and types of information processing. Additionally, future research can examine the moderating effects of fatigue, drowsiness and sleepiness (e.g., Hamid et al., 2016) on both trained and untrained drivers' AM behavior, specifically their sequential glance behavior. 
To sum, the current study reveals that the attention maintenance component of the SAFE-T training program can reduce glance durations most markedly on early glances in a sequence, especially the first glance where orienting of attention is most likely to occur.. Furthermore, effective countermeasures may also be designed and evaluated for both engineering (pavement markings, signal timing) and education (training, feedback) to reduce the proportion of in-vehicle glances greater than $2 \mathrm{~s}$, especially later in a glance sequence These findings may have implications for federal policies on driver distraction.

\section{ACKNOWLEDGEMENTS}

This research was supported by Summer Faculty Research Program, Office of Research, Old Dominion University awarded to Y.Y and Post-Doctoral Research Scholarship (2219), The Scientific and Technological Research Council of Turkey (TUBITAK) awarded to P. B.

\section{REFERENCES}

Bjørnskau, T., \& Sagberg, F. (2005). What Do Novice Drivers Learn during the First Months of Driving? Improved Handling Skills or Improved Road User Interaction? In G. Underwood (Ed.) Traffic and Transport Psychology: Theory and Application. (pp. 129-140). Amsterdam: Elsevier, B. V.

Chan, E., Pradhan, A.K., Pollatsek, A., Knodler, M.A., \& Fisher, D.L. (2010). Are driving simulators effective tools for evaluating novice drivers' hazard anticipation, speed management, and attention maintenance skills? Transportation Research Part F: Traffic Psychology and Behaviour, 13, 343353.

Divekar, G., Pradhan, A. K., Masserang, K. M., Reagan, I., Pollatsek, A., \& Fisher, D. L. (2013). A simulator evaluation of the effects of attention maintenance training on glance distributions of younger novice drivers inside and outside the vehicle. Transportation Research Part F: Traffic Psychology and Behaviour, 20, 154-169.

Divekar, G., Samuel, S., Pollatsek, A., Thomas, F. D., Korbelak, K., Blomberg, R. D., \& Fisher, D. L. (2016). Effects of a PC-Based Attention Maintenance Training Program on Driver Behavior Last Up to Four Months: A Simulator Study. In Transportation Research Board 95th Annual Meeting (No. 16-5667).

Drews, F. A., \& Strayer, D. L. (2008). Cellular phones and driver distraction. In M. A. Regan, J. D. Lee, \& K. L. Young (Eds.), Driver distraction: Theory, effects, and mitigation (pp. 169-190). Boca Raton, FL: CRC Press.

Green, P. (2002). Where do drivers look while driving (and for how long)? In R. E. Dewar \& P. L. Olson (Eds.), Human factors in traffic safety (pp. 77-110). Tucson, AZ: Lawyers and Judges.

Hamid, M., Samuel, S., Borowsky, A., Horrey, W. J., \& Fisher, D. L. (2016). Evaluation of training interventions to mitigate effects of fatigue and sleepiness on driving performance.

In Transportation Research Board 95th Annual Meeting (No. 16-3796).

Hedlund, J., Simpson, H., \& Mayhew, D. (2005). International Conference on Distracting Driving: Summary of proceedings and recommendations, Toronto, Canada, October 2-5. Ottawa, Ontario, Canada: Traffic Injury Research Foundation/Canadian Automobile Association.

Horrey, W. J. (2009). On allocating the eyes: Visual attention and in-vehicle technologies. In C. Castro (Ed.), Human factors of visual and cognitive performance in driving (pp. 151-166). Boca Raton, FL: CRC Press. 
Horrey, W. J., Wickens, C. D., \& Consalus, K. P. (2006). Modeling drivers' visual attention allocation while interacting with in-vehicle technologies. Journal of Experimental Psychology: Applied, 12, 67.

Klauer, S. G., Dingus, T. A., Neale, V. L., Sudweeks, J. D., \& Ramsey, D. J. (2006). The impact of driver inattention on near-crash/crash risk: An analysis using the 100-Car Naturalistic Driving Study data (No. HS-810 594). Washington, DC: National Highway Traffic Safety Administration.

McDonald, C.C., \& Sommers, M.S. (2015). Teen drivers' perceptions of inattention and cell phone use while driving. Traffic Injury Prevention, 16, S52-S58.

McKnight, A. J. \& McKnight, A. S. (2003). Young novice drivers: careless or clueless? Accident Analysis and Prevention, 35, 921-925.

Muttart, J.W., Fisher, D.L., Pollatsek, A.P., 2014. Comparison of anticipatory glancing and risk mitigation of novice drivers and exemplary drivers when approaching intersections in a driving simulator. In: Presentation at the 93rd Transportation Research Board Annual Meeting, TRB. National Research Council, Washington, D.C.

Muttart, J.W., Fisher, D.L., Pollatsek, A.P., \& Marquard, J. (2013). Comparison of anticipatory glancing and risk mitigation of novice drivers and exemplary drivers when approaching curves. In: Proceedings of the Seventh International Driving Symposium on Human Factors in Driver Assessment, Training, and Vehicle Design. Public Policy Center, University of Iowa, Lake George, NY. Iowa City.

Pollatsek, A., Divekar, G., \& Fisher, D. L. (2013). New measure of inattentiveness to forward roadway. Transportation Research Record: Journal of the Transportation Research Board, 2018, $31-37$.

Pradhan, A. K., Divekar, G., Masserang, K., Romoser, M., Zafian, T., Blomberg, R. D., ... \& Fisher, D. L. (2011). The effects of focused attention training on the duration of novice drivers' glances inside the vehicle. Ergonomics, 54, 917-931.

Pradhan, A., Masserang, K. M., Divekar, G., Reagan, I., Thomas, F. D., Blomberg, R., ... \& Fisher, D. (2009). Attention maintenance in novice drivers: Assessment and training. In Proceedings of the International Driving Symposium on Human Factors in Driver Assessment, Training, and Vehicle Design (Vol. 2009, p. 349).

Pradhan, A. K., Masserang, K. M., Pollatsek, A., Divekar, G., Romoser, M., Reagan, I., \& Fisher, D. L. (2010). Training attention maintenance: A first step. Presented at 89th Annual Meeting of the Transportation Research Board, Washington, D.C.

Pradhan, A.K., Hammel, K.R., DeRamus, R., Pollatsek, A., Noyce, D.A. \& Fisher, D.L., (2005). Using eye movements to evaluate effects of driver age on risk perception in a driving simulator. Human Factors, 47, 840-852.

Schneider, W. and R. M. Shiffrin (1977). Controlled and automatic human information processing: I. Detection, search and attention. Psychological Review, 84, 1-66.

Shinar, D. (2007). Distraction and inattention. In Traffic Safety and Human Behavior (pp. 517-564). Amsterdam: Elsevier.

Summala, H., Lamble, D., \& Laakso, M. (1998). Driving experience and perception of the lead car's braking when looking at in-car targets. Accident Analysis and Prevention, 30, 401-407.

Yamani, Y., Horrey, W.J., Liang, Y., \& Fisher, D.L. (2015). Sequential in-vehicle glance distributions: an alternative approach towards analyzing glance data. Human Factors, 57, 567-572.

Yamani, Y., Samuel, S., Knodler, M. A., \& Fisher, D. L. (2016). Evaluation of the effectiveness of a multi-skill program for training younger drivers on higher cognitive skills. Applied Ergonomics, 52, 134-141. 